\title{
Design an Improved Hybrid Routing Protocol Strategy to Minimize Delay \& Overhead for MANET
}

\author{
Advin Manhar \\ JECRC UNIVERSITY, JAIPUR \\ adymanhar@gmail.com \\ Dr. Deepak Dembla \\ JECRC UNIVERSITY, JAIPUR \\ deepak.dembla@jecrcu.edu.in
}

\begin{abstract}
Mobile adhoc networks (MANET) are a self-coordinated wireless network that is worked without perpetual foundation and base station endorsement. In MANETs (Mobile Ad Hoc Networks) every single node activity goes about as the information source and communicator router. It notices its useful neighbours by making themselves by passing the nodes that got terminated of that correspondence range. In this paper, we designed the IHRP routing protocol with the help of combining the AODV, AOMDV and OLSR routing protocol with the network situation based routing adaptation. In the previous analysis, we identify AODV is more compatible for dynamic environment (where node motion is higher). The AOMDV routing protocol is useful for network load balancing and congestion control. OLSR is compatible for stable network which use as maximum link stability base routing. While we are designing the IHRP routing protocol through of the above (AODV, AOMDV and OLSR) protocol .We handle the network behaviour with better performance in any situation. Initially the RREP are broadcasted to find out the route. The poll of routing, select based on the network situation. The proposed IHRP start with a RREQ packet is to search the distance from its origin to its objective node. The network motion is higher than AODV protocol handle the particular situation but in any situation, while the 70\% network node are stable then the route selection and transferring of data with the help of an OLSR routing protocol. The propose IHRP routing protocol also handle the network load and control the network congestion through the AOMDV routing protocol. Our proposed protocol IHRP also works in a hybrid manner while the source and destination belong in longer range such as different cluster. At that time situation based routing was followed between the clusters. We saw in the result part the comparison of IHRP and ZRP routing protocol. Execution of the improved hybrid routing protocol (IHRP) is much better than hybrid routing protocol (ZRP). In case of Packet Delivery Ratio analysis for IHRP and ZRP, we clearly show that The Packet Delivery Ratio is $97.99 \%$ by IHRP and $79.49 \%$ by ZRP, so we can say that the performance of packet delivery ratio in Improved hybrid routing protocol (IHRP) is much better than ZRP, where Improved hybrid routing protocol (IHRP) and hybrid routing protocol (ZRP) both performed the conjunction of proactive and reactive routing protocol features therefore we can say that Packet Delivery Ratio is better in improved hybrid routing protocol (IHRP) as compare to hybrid routing protocol (ZRP).
\end{abstract}

We clearly show that the Normal Routing Load analysis for IHRP and ZRP where Normal Routing Load is $0.11 \%$ by IHRP and $4.72 \%$ by ZRP, so we can say that performance of Improved Hybrid Routing Protocol (IHRP) in case of a Normal Routing Load is much less than the Hybrid Routing Protocol (ZRP).

In case of overall parameter matrix (Normal Routing Load, Throughput, Packet Delivery Ratio and Average End To End Delay) the performance of Improved Hybrid Routing Protocol (IHRP) is much better than existing Hybrid routing protocol (ZRP).

Keywords: MANET, IHRP, AODV, ZRP, AOMDV, OLSR, NS-2.

\section{Introduction}

The Mobile adhoc networks (MANET) is a wireless network that is self-coordinated worked without perpetual foundation and base station endorsement. In Mobile adhoc networks for sending \& receiving of data packets wireless surface is used that is consists of mobile nodes. Ad- hoc networks [16] linked remotely with a self 
designed network without having a fixed foundation consisting of a set of mobile nodes. Adhoc network mostly used by the labourers in a hazardous situation, soldiers for enemy territory or a gathering of chiefs at an outside area. Figure 1 shows an exemplary MANET. In a customary fixed structure network, direct need to pass on with from everyone others needs to initially connect to the closest base station that sends their solicitations to the base station that is nearest to the target nodes. In Mobile adhoc networks (MANETs), every one of these errands is performed by the actual nodes themselves.

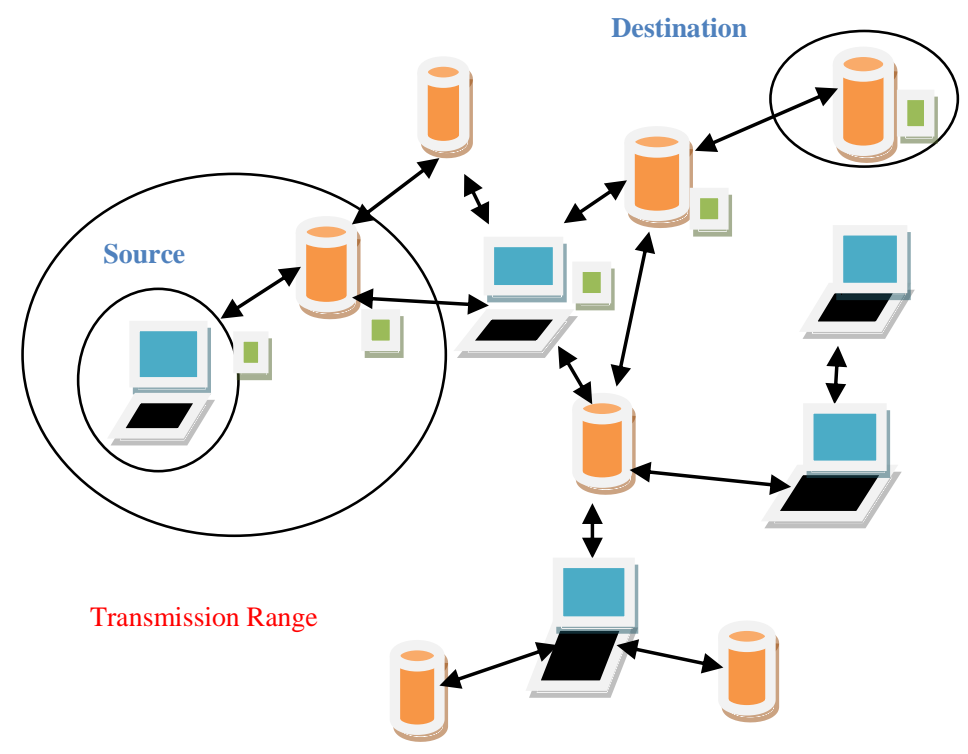

Figure No. 1 Mobile Ad-Hoc Network (MANET)

\section{Routing Protocols}

\section{A: Table Driven (Proactive) Routing Protocol}

Table driven routing protocols are also called as Proactive routing protocols. Proactive routing protocols kept the routing table information of every single node. In Table driving routing protocols the entire nodes transfer data packets based on the predefined route in the routing table. In Table driven routing protocols, the entire path has to be predefined before forwarding the data and the minimum delay and routing overhead is maximum in case of data packet transferring. For Example: Wireless Routing Protocol and Destination sequence distance vector [8] etc.

\section{Destination-Sequenced-Distance-Vector Routing}

It is the table-driven routing protocol. Distance Vector Routing is one of the two kinds of routing protocols where every router informs its neighbour regarding its routing table. Each and every single node in the network keeps the routing table in which transferring the data from origin to target nodes are recorded by the number of hops. Entire entry in DSDV is recognized by the sequence number selected by the target node. In Distance Vector Routing it maintains the consistency with the help of Routing table.

\section{B: Reactive (On-Demand) Routing Protocol}

In On-Demand Routing Protocol there is a route discovery method that is used where if sender node wants to transmit the data to the receiver target node, and then it will send the RREQ packet to the network, if destinations are found, and then it will establish the connection and transmit the data. There are different types of reactive routing protocols: DSR (Dynamic Source Routing), AODV (Ad-hoc On-Demand Distance Vector routing). 


\section{Adhoc On-Demand Distance Vector (AODV)}

It is an important on-demand routing protocol. In the case of AODV [21], for every new communication, the route discovery process will be started from the beginning (starting node) for this reason AODV routing protocol consumes more routing over-head and bandwidth.

\section{C: Hybrid Routing Protocol}

The hybrid routing protocol is performed by the conjunction of proactive and reactive routing protocol features. HRP is used for the zone concept for the routing so the routes found it quickly. For Example: - ZRP (Zone routing protocol).

\section{Zone Routing Protocol (ZRP)}

The Zone routing protocol is the grouping of proactive and reactive routing protocols $[6,1]$. The main concept of a Zone routing protocol is Intra-zone routing protocol (IARP) and inter-zone routing protocol (IERP). IARP used inside the zone and here we use proactive routing protocol and in the case of IERP, it is used outside the zone and here we use the reactive routing concept.

\section{Related Work}

In recent past year the Independent Zone Routing Protocol (IZRP) proposed by the Dilli Ravilla et.al. The IZRP implements the distribution and adaptation product for all the node traffic as per strength of each node. Here IZRP and ZRP operation performed by the author with respect to PDF, Delay and NRL [1].

Hybrid routing protocol with Broadcast Reply (HRP-BR) scheme presented by the P. M. Jawandhiya et.al in 2010. The author proposed a protocol scheme that has a quality to reduce the number of RREQ, delay and RREP packets broadcasted on the network and also support to minimizing the energy level of nodes because the antennas (transmitter and receiver) will not waste their energy in R/C-TS signalling. For broadcast packets, there is no need of RTS-CTS transmission that is another advantage of this proposed protocol. HRP- Broadcast Reply is weighed with the prevailing A-O-D-V which shows the result of HRP- Broadcast Reply increases the PDR and reduction in delay and minimizes the overhead of routing [2].

Mrs. K. Kavitha et.al in 2013, presented a Robust and Scalable protocol (RSGM). In RSGM the data packets and control communication are fore along cost-effective tree. RSGM, ODMRP and SPBM are analyzed with the operating parameter like control overhead, average joining delay, average path length and packet delivery ratio by unstable network ranges, group size, node density and moving speed [3].

Pardeep Singh and Amit Jaiswal in 2010, Introduced an Optimizing Velocity Based Adaptive Zone Routing Protocol (OVBAZRP). Performance of OVBAZRP is much better in the case of firm routing where node speed is not in regular format. An OVBAZRP is proposed where different nodes select various zone regions consistently; the IARP and IERP of ZRP are configured. Optimizing Velocity Based Adaptive ZRP modifies the zone routing protocol and movement of the nodes during a MANET are often determined within an inexpensive computation. There are 3 concepts used in ZRP i.e. BRP, IARP and IERP. The main concept is that the radiuses of every node are based on the approaching and mobility design. The reactive routing protocols (TORA, DSR) used as IERP, BRP used for managing the flooding by packets and table driven routing protocol (DSDV) used as IARP [4].

In 2007, proposed a new concept, i.e. Velocity Based Adaptive-ZRP (VBAZRP) by Jieying Zhou et.al which allows different types of nodes to decide various zone regions apropos to each one node's chiselled mobility. Velocity Based Adaptive ZRP redesigns the protocols of the Zone Routing Protocol, and designed a new Velocity Based Adaptive-IA which purpose irregular message and reply concept and regular common update 
concept to keep node's proactively communication in the zone. The experimental results show that VBAZRP can change better in different scenarios to other ad hoc enforcements [5].

Shailendra Kumar Pathak et. al. in 2014 introduced an algorithm for query packets and gave the concept about how to control the query packets in the network. Here the author used network simulator Qualnet 5.0.2 for obtaining results and after the final result the performance has been increased [6].

Abhishek purohit and gajendra singh chandel in 2012 protocols in mobile adhoc when node density changes. There are different types of routing protocol in mobile adhoc network described the operations of various types of network routing like Destination-Sequenced Distance Vector (DSDV), Optimized Link State Routing (OLSR) and Ad Hoc On-demand Distance Vector (AODV), Dynamic Source Routing (DSR), each and every MANET protocol operation in the network has its own disadvantages and advantages. With the help of NS-2 software, they calculated different metrics: packet drop, packet delivery ratio, and routing load [7], end-to-end delay.

Rosilah and Adam in 2013 gave an overview for QoS in reactive routing protocols and focused on delay on a network. The primary intention of Mobile Adhoc Networks is Quality of Service routing to provide a path between different nodes. Prompt QoS is used in mainly real-time transaction and multimedia systems. The most on-demand routing protocols used in MANETs are AODV and DSR. AODV and DSR protocols use the least lane to set up routing between source and destination [8].

Sheng Liu et. al. In 2013 described the main techniques of MANETs, and compared the reactive and proactive routing protocol systems, When DSR, AODV, TORA and ABR comes inside the reactive routing protocol for MANET. Here the author introduces in concise the Adhoc on Demand Vector routing protocol. They presented a reform protocol: B-AODV, supported on the routing repair of AODV and lack of routing detection. The simulation result is based on NS2 and incitement to the operation of AODV and B-AODV. The resulting scenario of B-AODV is more desirable than the AODV [9].

In 2019 by Aastha Mishra et.al. discussed different routing protocols in MANETs, and also generated results of different routing protocols like ZRP, DSR, FSR, AODV, DSDV, OLSR TORA and AODV. A better performance routing protocol in the result part is DSR and AODV protocols as compared to other protocols. TORA and DSDV performance have no better results. Reactive protocols are more suitable when node moments are frequent and proactive protocols are more suitable when node moments are infrequent so the solution can be a hybrid routing protocol [10].

Hassan al-mahdi and hasssn shaban in 2016, introduced a QCS-AODV (Queue Congestion Status AODV) routing protocol. The QCS-AODV is an adapted variant of the present AODV (Adhoc on Demand Vector) protocol. The QCS-AODV mainly used for path selection is queue congestion and hop counts. They also introduced a PQM (partition queue management) concept to handle node queues. Here the author used network simulator NS2 for obtaining results in different parameter (packet dropping and delay) and after the final result the network performance has been increased in MANET by using partition -queue- management concept and QCS-AODV (Queue Congestion Status AODV) routing protocol [11].

B. Basaveswara Rao et.al. In 2018 investigated the effect of mobility patterns in nodes on different routing protocols in MANET by using NS-2. The technique report is carried out in two strategies and they are grouped founded on framing accessibility at nodes. They are (1) a limited compound is created for each one node called Buffer Node (BN Strategy) and (2) buffer facility is not available at each node called Buffer Less Node (BLN Scheme). In this simulation model is reasoned with, quality parameters are speed and pause times are considered for reactive routing protocols [12].

Amar Singh and Sagheer Ahmed in 2016 provided the Literature analysis for hybrid, reactive and proactive routing protocols in Mobile Adhoc Network. They also perform the comparison of them, where different routing protocols have different types of advantages and disadvantages depending upon the network situation. Mobile adhoc networks (MANET) are a self-coordinated wireless network that is worked without perpetual foundation 
and base station endorsement. In MANETs (Mobile Ad Hoc Networks) every single node activity goes about as the information source and communicator router [13].

Fahad Taha AL-Dhief et.al. In 2018 He presented the comparative performance between reactive and proactive routing protocol where routing protocols are ad hoc on-demand distance Vector Routing and DSDV and Dynamic Source Routing. The simulation result generated on the basis of E-to-E delay and PDR. The result shows that the performance of AODV with respect to PDR is desirable than DSR and DSDV [14].

H A Sidharta et.al. in 2018, performed a relative study of DSR routing protocols and DSDV routing protocol for MANATs. The simulation result generated on the basis of Dropped Packets, PDR, Throughput and E-to-E delay and the result shows that the function of DSDV is more desirable than DSR routing protocol [15].

\section{Proposed Work and methodology}

The main challenges in MANET routing protocol implementation are how to maintain the random movement of nodes and maintain the continued changes topology of the network. The previous resulting analysis part is the comparison of DSDV, ZRP and AODV routing protocol. In comparison with AODV and DSDV routing protocols, ZRP has a more desirable performance. Here we design the IHRP routing protocol with the help of combining the AODV, AOMDV and OLSR routing protocol with the network situation based routing adaptation. In the previous analysis, we identify AODV is more compatible for a dynamic environment (where node motion is higher).The AOMDV routing protocol is useful for network load balancing and congestion control. OLSR is compatible for stable networks which use maximum link stability based routing. While we are designing the IHRP routing protocol through the above protocol we handle the network behaviour with better performance in any situation. The proposed IHRP is a mixture of reactive and proactive routing protocol where node movement is frequent or infrequent the performance of this new improved proposed routing protocol (IHRP) maintains the network performance. The IHRP will definitely minimize the data re-transmissions of nodes, delay, routing load (RL) and maximize the packet delivery ratio (PDR), throughput and data transmission rate of the network as compared to the existing hybrid routing protocol in MANET. We discussed here the performance of the two routing protocols i.e. proposed IHRP and ZRP and Mobile Adhoc Network. On the basis of their simulation results, the performance of IHRP is better than ZRP. By using this protocol (IHRP) we can also beat the energy utilization issues in mobile ad hoc networks.

\subsection{Working of IHRP:}

IHRP is an Improved Hybrid Routing Protocol. The concept of IHRP is to tap the quality of reactive and proactive routing protocol.

We can avoid the flooding process and minimize the time if we use the characteristics of pro-active and re-active protocol techniques for the route discovery process. Process of flooding makes the unpredictable network and increases the data packet dropping. If we want to conquer the above flooding problem, the process of route discovery should be completed in less time. So we designed an IHRP routing protocol with the help of combining the AODV, AOMDV and OLSR routing protocol with the network situation based routing adaptation. In the previous analysis, we identify AODV is more compatible for a dynamic environment (where node motion is higher). Our proposed protocol is also based on the energy consumption scenario.

The AOMDV routing protocol is useful for network load balancing and congestion control. OLSR is compatible for stable networks which use maximum link stability based routing. While we are designing the IHRP routing protocol through the above protocol (AODV, AOMDV and OLSR) we handle the network behaviour with better performance in any situation.

Initially the route request packet is broadcasted to search the path from origin to target node. The selection of routing protocol totally based on the network situation. The proposed IHRP initiated with a RREQ packet to search the path from origin to target node and if the network node movement is higher than $10 \mathrm{M} / \mathrm{Sec}$, AODV protocol handles the particular situation and broadcasts the RREQ packet, and select the shortest path between the communicator node, but any situation, while the network node movement is less than $10 \mathrm{M} / \mathrm{Sec}$, the 
route selection and transferring of data with the help of an OLSR routing protocol. The proposed IHRP routing protocol also handles the network load and controls the network congestion through the AOMDV routing protocol.

If any established communication is detected as higher load in the network (higher utilization of resources) than multiple paths are created to handle or balance the network load and increase the network performance. The proposed IHRP is a combination of reactive and proactive protocol where node movement is frequent or infrequent the performance of this new proposed improved routing protocol maintains the network performance. The IHRP will definitely minimize the amount of data re-transmissions in the network, NRL and E-to-E delay and also maximize the packet delivery ratio (PDR), data transmission rate and throughput of the network as compared to obtainable hybrid routing protocol in MANET.

The proposed Improved-Hybrid-Routing-Protocol (IHRP) is based on this concept where the Route Request Packet (RREQ) is sent by the sender to find the destination (D) in the network. If the sender (S) finds the destination (D) then store the node movement, speed of node movement, the energy of node and also store the direction of moving node. If the sender did not find the destination, then Continuous sand the RREQ packet through neighbor node until the destination is not found, then after store the node movement, speed of node movement, energy of node and also store the direction of moving node. After the storing of node information, sender (S) node will move to the selection of routing protocol (AODV or OLSR). The selection of routing protocol is based on node movement, if the node movement is greater than 10 meters/second (its means the chances of node participation in the network is not longer) so their selection of routing protocol is AODV because AODV has a quality to handle this type of condition. The RREP packet send by the destination node to the sender node after the selection of routing protocol, then the connection will be established between sender and receiver. After the connection established, It will move to the single path route decision and check the congestion in the node, If congestion in the node then it will be resolve by the AOMDV routing protocol and send the data from origin to target and finish the transmission as well straight transmit the data packet from source to target and stop the transmission. The routing protocol selection is totally based on node movement, if the node movement is less than 10 meters/second (its means the chances of node participation in the network is higher) so their selection of routing protocol is OLSR where OLSR is a proactive routing protocol. The target node sends the RREP packet to the origin after the routing protocol selection then the connection will be established between sender and receiver, It will move to the single path route decision and check the congestion in the node, If congestion in the node then it will be resolve by the AOMDV routing protocol and transmit the data from source to destination and end the transmission as well straight transmit the data packet from source to target and stop the transmission.

Our execution environment is based on:

\section{Packet delivery ratio:}

In the case of PDR where we calculate the ratio of the actual packets accepted at the target nodes and the total packets sent by the origin node.

\section{End to End Delay:}

In the case of E-to-E delay, it calculates the overall delay where the time of the data packet was sent by the origin is compared to the time it was received at the destination.

Routing load: Here we calculate how much supporting control packets delivered at the destination for every data packet.

\section{Throughput:}

Here we calculate per second how much packets send to the target node. 


\section{Proposed Flow Chart for IHRP}
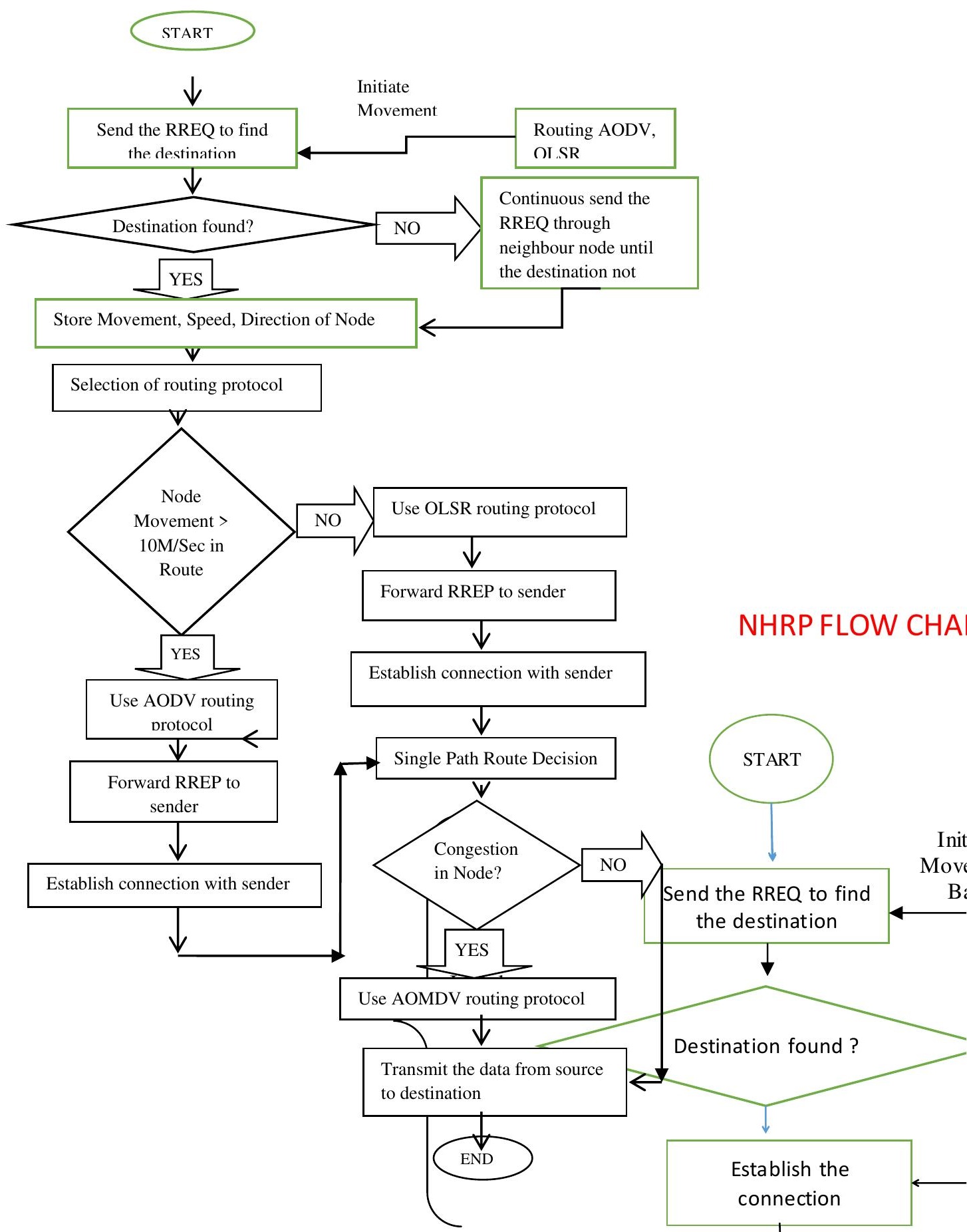

Figure No. 2 Proposed Flow Chart for IHRP

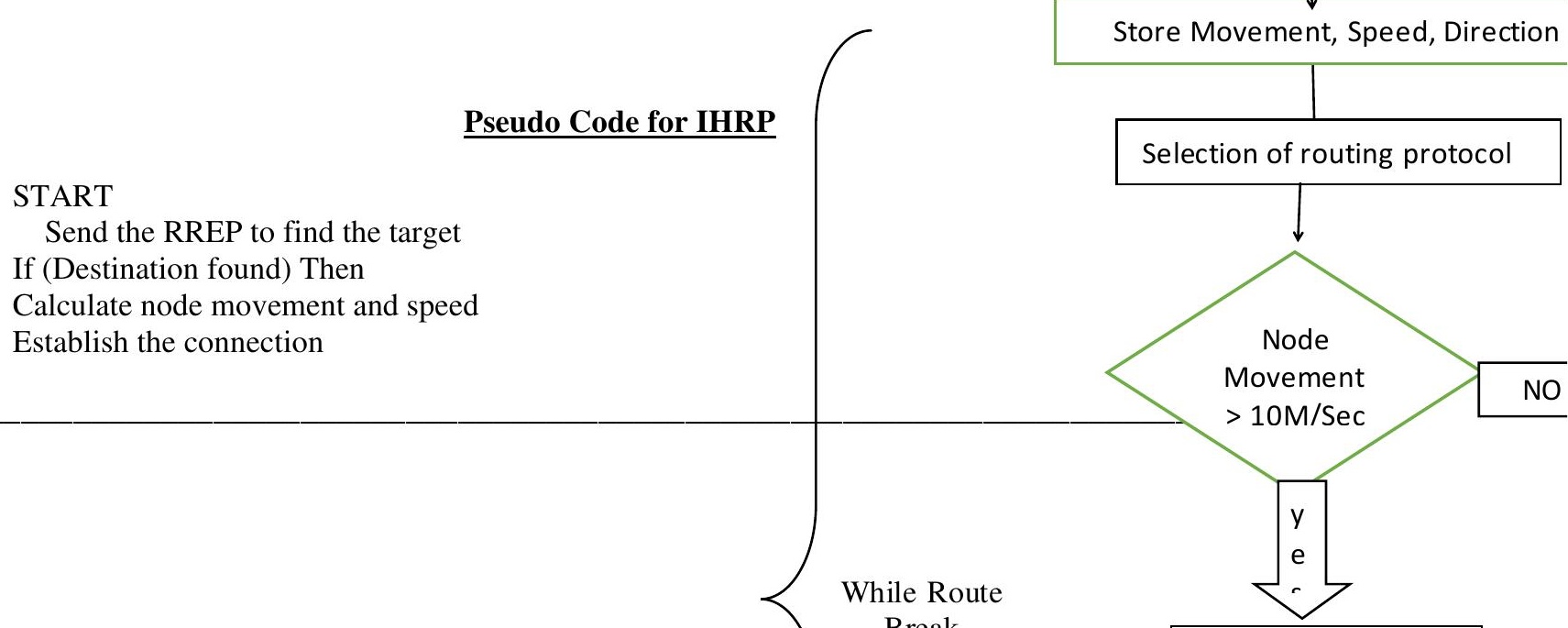


If (Node Mobility > 70\%) Then

Use AODV routing protocol

If (Congestion in Node) Then

Use AOMDV routing protocol

Spread the information Origin to target

End If

Else

Spread the information Origin to destination

End If

If (Node Mobility <=70\%) Then

Use OLSR routing protocol

If (Congestion in Node) Then

Use AOMDV routing protocol

Transmit the data from source to destination

End If

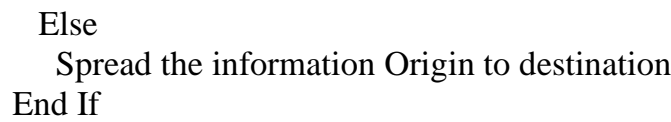

Else

Continuous send the RREQ through neighbour node until the destination not found

End If

\section{Simulation Environment}

The entire simulations were done by utilizing a network simulator (NS) 2.31. The purpose of Network Simulator-2 is to endorse education and research. Network Simulator is appropriate for traffic evaluations, comparing different protocols and novel protocols designing. Network Simulator-2 is open-source software. NS2 used by the researchers and a large number of institutes. Network Simulator-2 Versions are available for Windows, Linux, Mac OS X and Solaris [22].

\subsection{Simulation Parameters:}

Results have been generated according to the below given parameters.

TABLE I

Simulation PARAMETERS

1. Simulation Parameters

\begin{tabular}{|l|l|}
\hline Parameters & Configuration Value \\
\hline Routing Protocol & IHRP, ZRP \\
\hline Simulation Area & $800 * 800$ \\
\hline Network Type & MANET \\
\hline Nodes/Devices & 25 \\
\hline Physical Medium & Wireless \\
\hline Node Movement & Random \\
\hline
\end{tabular}




\begin{tabular}{|l|l|}
\hline Simulation Iteration & 100 \\
\hline Queue Length & 10 \\
\hline MAC Layer & MAC 802.11 \\
\hline Traffic Type & CBR, FTP \\
\hline Propagation radio model & Two ray ground \\
& \\
\hline Rate & Random \\
\hline
\end{tabular}

Table No: 1 Simulation Parameters

\subsection{Rresults}

In result part we present simulation results of IHRP and ZRP routing protocol.

\section{Packet Delivery Ratio Analysis:}

The figure no. 3 represents the PDR analysis for IHRP and ZRP. Here we clearly show that The PDR is 97.99 $\%$ by IHRP and $79.49 \%$ by ZRP, so we can say that execution of the improved hybrid routing protocol (IHRP) in case of packet delivery ratio is far higher than the ZRP.

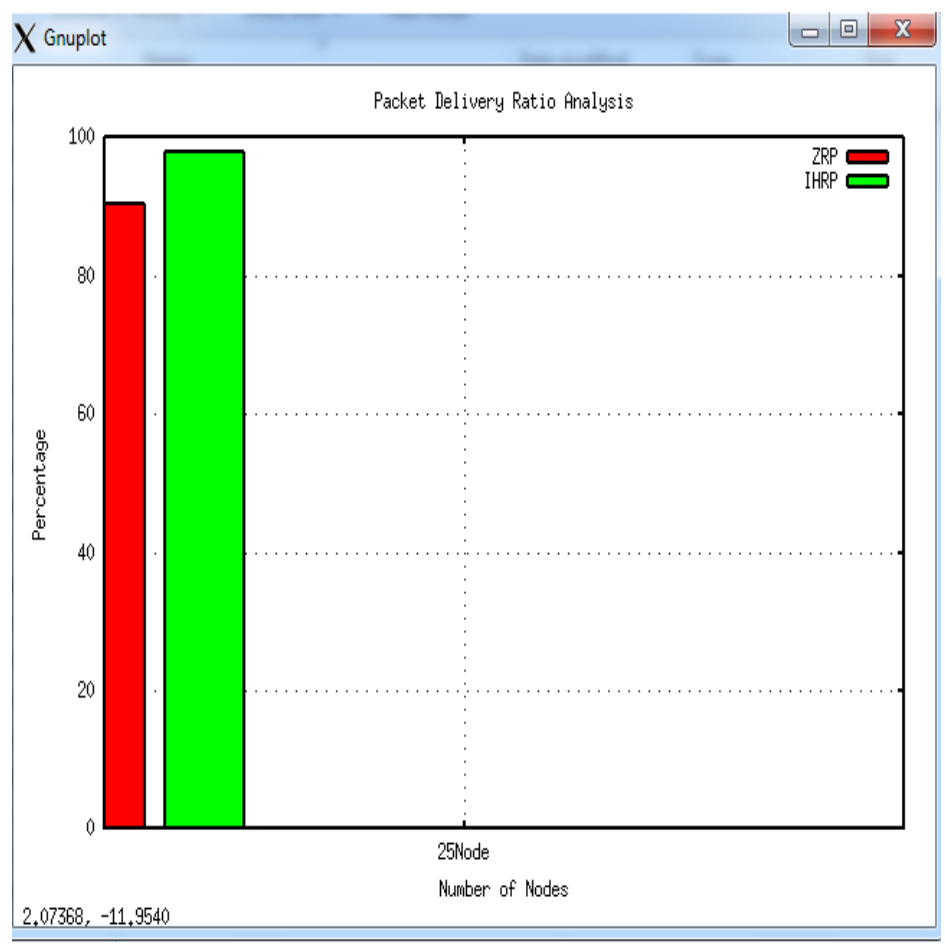

Figure No 3: "Packet Delivery Ratio" Analysis for IHRP and ZRP 


\section{Throughput Analysis:}

The figure no. 4 represents the Throughput analysis for IHRP and ZRP. Here we clearly show that The Throughput is 3.53 by IHRP and 2.48 by ZRP, so we can say that performance of Improved Hybrid Routing Protocol (IHRP) in case of Throughput is much better than ZRP.

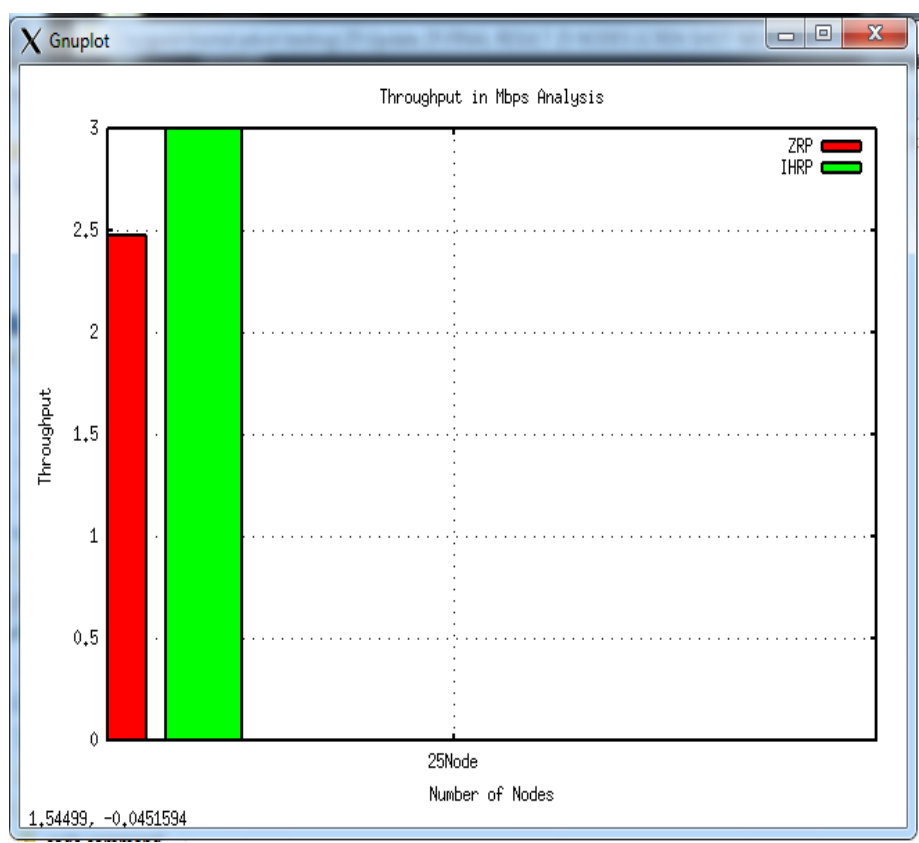

Figure No 4: "Throughput” Analysis for IHRP and ZRP

3. Normal Routing Load:

The figure no. 5 represents the Normal Routing Load analysis for IHRP and ZRP. Here we clearly show that The Normal Routing Load is $0.11 \%$ by IHRP and $4.72 \%$ by ZRP, so we can say that performance of the Improved Hybrid Routing Protocol (IHRP) in case of a Normal Routing Load is much less than Hybrid Routing Protocol (ZRP).

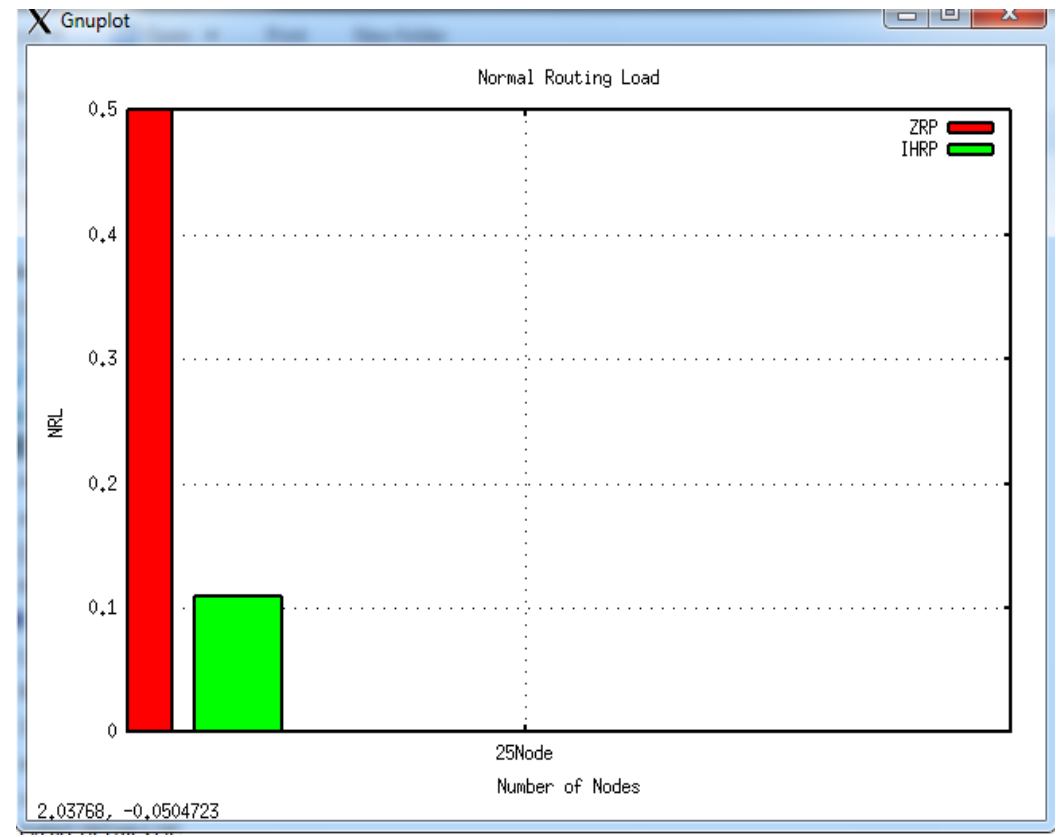

Figure No 5: "Normal Routing Load" Analysis for IHRP and ZRP 
4. Average End to End Delay (ms) Analysis:

The figure no. 6 represents the analysis of Average E-to-E Delay for IHRP and ZRP. Here we clearly show that The Average E-to-E Delay is 0.04 by IHRP and 0.21 by ZRP, so we can say that the performance of the improved hybrid routing protocol (IHRP) in case of Average E-to-E Delay is very less than (ZRP) Hybrid routing protocol.

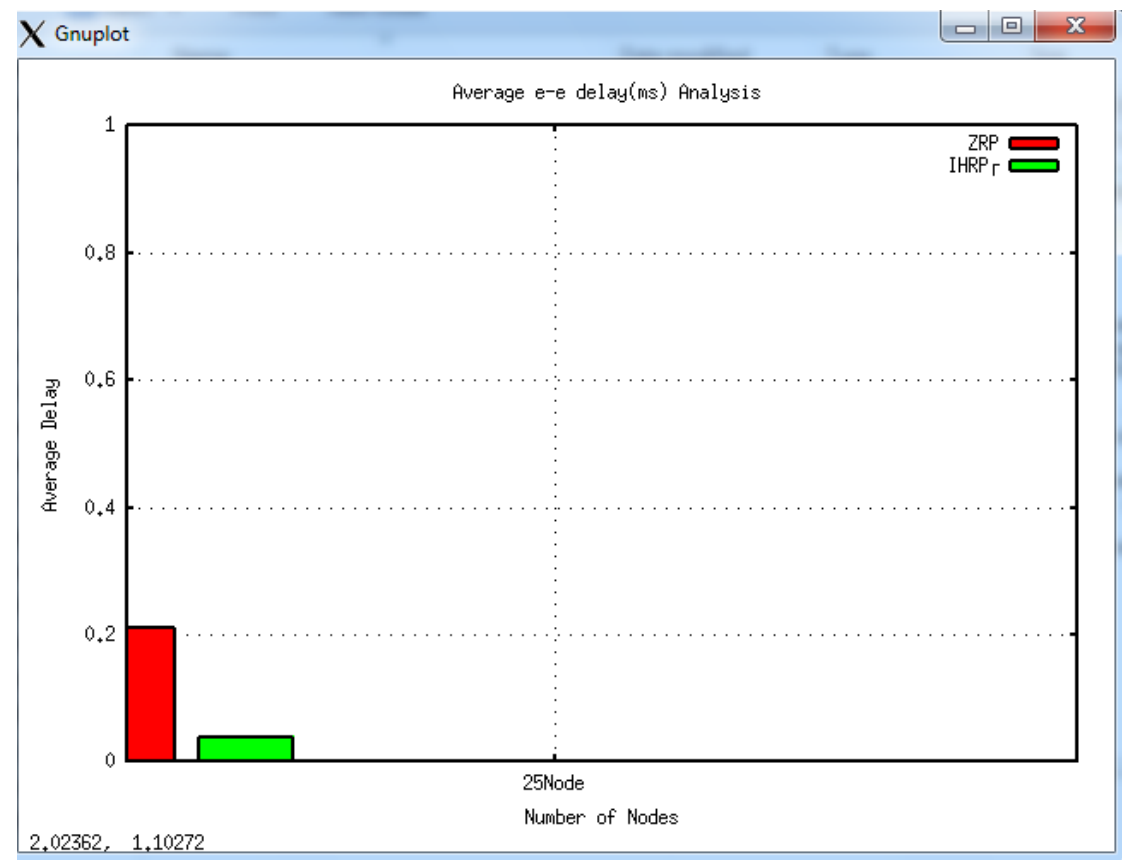

Figure No. 6: “Average End to End Delay" Analysis for IHRP and ZRP

\subsection{Performance Matric Ssummary}

The whole execution of IHRP and ZRP protocols is based on below given metric.

TABLE II: Performance Metric Results

\begin{tabular}{|l|l|l|}
\hline Parameters & IHRP & ZRP \\
\hline PDR & $\mathbf{9 7 . 9 9}$ & $\mathbf{7 9 . 4 9}$ \\
\hline THROUGHPUT & 3.53 & 2.48 \\
\hline NRL & $\mathbf{0 . 1 1}$ & $\mathbf{4 . 7 2}$ \\
\hline E-E- DELAY & $\mathbf{0 . 0 4}$ & $\mathbf{0 . 2 1}$ \\
\hline
\end{tabular}


In above performance metric we show that the Packet Delivery Ratio is $97.99 \%$ by IHRP and $79.49 \%$ by ZRP, hence the performance of the improved hybrid routing protocol (IHRP) in the case of PDR is much better than ZRP.

The Normal Routing Load is $0.11 \%$ by IHRP and $4.72 \%$ by ZRP, so the operation of the Improved HRP in case of a Normal Routing Load is much less than Hybrid Routing Protocol (ZRP).

We show in the result that the Throughput is 3.53 by IHRP and 2.48 by ZRP; So on the basis of performance the Throughput of the IHRP is much better than ZRP.

In the above performance metric of Average End to End Delay for IHRP and ZRP where Delay is 0.04 by IHRP and 0.21 by ZRP. Here we clearly show that the result of the IHRP in the case of Average E-to-End Delay is less than ZRP.

The overall parameter matrix the result of the Improved Hybrid Routing Protocol (IHRP) is far higher than the existing Hybrid routing protocol (ZRP).

\section{Conclusion and Future Work}

The Mobile adhoc networks (MANET) is a wireless network that is self-coordinated worked without perpetual foundation and base station endorsement. In MANETs (Mobile Ad Hoc Networks) every single node activity acts as an information source and communicator router. It notices its effective neighbors and with the support of them, it will converse to the nodes that are terminated of its correspondence range. The whole simulations were done by utilizing NS 2.31 network simulator and performing the operation on IHRP and ZRP routing protocols. We proposed an improved hybrid routing protocol called IHRP. IHRP is a combination of reactive and proactive routing protocol as an existing ZRP. In the case of Improved Hybrid Routing Protocol (IHRP) included inside the proactive routing protocol is OLSR and the reactive protocols are AOMDV and AODV. With the help of these three routing protocols (AOMD, AODV and OLSR) we designed an improved hybrid routing protocol IHRP.

\section{References}

3. Dilli Ravilla,V.Sumalatha and Dr Chandra Shekar Reddy Putta, (December 2011). Hybrid routing protocols for ad hoc wireless networks, International Journal of Ad hoc, Sensor \& Ubiquitous Computing (IJASUC) Vol.2, and No.4.

4. P. M. Jawandhiya, R. S. Mangrulkar and Mohammad Atique,(October -2010). A novel hybrid routing protocol for mobile adhoc network, International Journal of Advancements in Technology( IJoAT), ISSN 0976-4860, Vol 1, No 2.

5. Mrs.K.Kavithal ,Dr.K.Selvakumar ,Mrs.T.Nithya and Ms.S.Sathyabama, (2013). Zone based multicast routing protocol for mobile ad-hoc network, IEEE, 978-1-4673-5301-4/13/\$31.00

6. Amit Kumar Jaiswal, Pardeep Singh, (2010). Optimizing velocity based adaptive zone routing protocol, IEEE- Int'l Conf. on Computer \& Communication Technology (ICCC).

7. Jieying Zhou, Yuliang Cheng, Jianlin Lu, (2007). Velocity based adaptive zone routing protocol, Proceedings of 2007 International Symposium on Intelligent Signal Processing and Communication Systems, IEEE-Nov.28-Dec.1, Xiamen, China.

8. Shailendra Kumar Pathak, Raksha Upadhyay, Uma Rathore Bhatt, (2014). An efficient query packets forward algorithm in zrp protocol, IEEE - 978-1-4799-2900-9/14.

9. Abhishek purohit,gajendra singh chandel, (2012). Analysis of AODV and DSDV routing protocol in MANET, International Journal of Electronics Communication and Computer Engineering (IJECCE), Volume 4, Issue 2, ISSN (Online): 2249-071X, ISSN (Print): 2278-4209.

10. Saad M. Adam,Rosilah Hassan,( 2013). Delay aware reactive routing protocols for QoS in MANETs: a Review, Journal of Applied Research and Technology, Vol. 11.

11. Sheng Liu, Yang Yang, Weixing Wang, (2013). Research of AODV routing protocol for ad hoc networks, ELSEVIER-AASRI Conference on Parallel and Distributed Computing and Systems, AASRI Procedia 5, p.no. $21-31$.

12. Aastha Mishra,Shweta Singh,Arun Kumar Tripathi, (2019). Comparison of MANET routing protocols, A Monthly Journal of Computer Science and Information Technology (IJCSMC), Vol. 8, Issue. 2, ISSN 2320-088X,pg.67- 74

13. Hassan al-mahdi, hasssn shaban, (2016). Performance analysis of queue congestion status Routing protocol (qcs-aodv) for ad hoc networks, International Journal of Advanced Computational Engineering and Networking, ISSN: 2320-2106, Volume-4, Issue-2. 
14. B.Basaveswara Rao, SK. Meera Sharief K. GangadharRao, (2018). Impact of Mobility on Routing Protocols in MANET using NS2, International Journal of Scientific Research in Computer Science, Engineering and Information Technology (IJSRCSEIT), Volume 3, Issue 5, ISSN: 2456-3307.

15. Sagheer ahmed, amar singh, (2016). Literature survey of manets routing protocols, International Journal Of Technology and Computing (Ijtc), issn-2455-099x, volume 2, issue 7.

16. Fahad Taha AL-Dhief, Naseer Sabri , M.S. Salim, S. Fouad, S. A. Aljunid, (2018). MANET Routing Protocols Evaluation: AODV, DSR and DSDV Perspective, MATEC Web of Conferences 150, 06024.

17. H A Sidharta, Sidharta, C Huda, (2017). Evaluation QOS and energy consumption for DSDV and DSR in MANET (Mobile ad hoc network), 1st International Conference on Engineering and Applied Technology (ICEAT),IOP Conf. Series: Materials Science and Engineering 403 ,012058 doi:10.1088/1757-899X/403/1/012058.

18. J.-E. Garcia, A. Kallel, K. Kyamakya, K. Jobmann, J.-C. Cano, P. Manzoni, (2003). A novel dsr-based energy-efficient routing algorithm for mobile ad-hoc networks, IEEE Vehicular Technology Conference, VTC Fall, Orlando, USA, Vol. 5, pp. 2849 - 2854, 6-9.

19. Christopher N. Ververidis, George C. Polyzos, (2005). Routing layer support for service discovery in mobile ad hoc networks, PERCOMW, Third IEEE International Conference on Pervasive Computing and Communications Workshops (PERCOMW'05), pp. 258-262.

20. V. Venugopal, R. Bartoš, M.J. Carter, and S.S. Mupparapu, (2003). Improvement of robustness for ad hoc networks through energy-aware routing, Proc. of the Fifteenth IASTED International Conference on Parallel and Distributed Computing and Systems (PDCS), Marina del Rey, CA, (T. Gonzales ed.).

21. W. Yu and J. Lee, (2002). DSR-based energy-aware routing protocols in ad hoc networks, Proceedings of the Int. Conf. on Wireless Networks (ICWN), Las Vegas, Nevada.

22. D. Kim, J.J. Garcia-Luna-Aceves, K. Obraczka, J, Cano, and P. Manzoni, (2002). Power-aware routing based on the energy drain rate for mobile ad hoc networks, 11th IEEE International Conference on Computer Communication and Networks. pp.565- 569.

23. S.K. Das, A. Mukherjee, et al, (2003). An adaptive framework for QoS routing through multiple paths in ad hoc wireless newtorks", J. Parallel Distributed Computing, pp. 141-153.

24. Fast NS-2 simulator website http://lst.inf.ethz.ch/fast-ns2/. 